\title{
Improving care through health economics analyses: cost of illness and headache
}

\author{
Francesco Saverio Mennini · Lara Gitto • \\ Paolo Martelletti
}

Received: 1 February 2008/ Accepted: 10 June 2008/Published online: 5 July 2008

(C) Springer-Verlag 2008

\begin{abstract}
The impact of headache disorders is a problem of enormous proportions, both for individual and society. The medical literature tried to assess its effects on individuals, by examining prevalence, distribution, attack frequency and duration, and headache-related disability, as well as effects on society, looking at the socio-economic burden of headache disorders [Rasmussen (Cephalalgia 19:20-23, 1999)]; [Lanteri-Minet et al. (Pain 102:143-149, 2003)]. The issue of costs represents an important problem too, concerning both direct and indirect costs. Direct costs concern mainly expenses for drugs. Migraine has a considerable impact on functional capacity, resulting in disrupted work and social activities: many migraineurs do not seek medical attention because they have not been accurately diagnosed by a physician or do not use prescribed medication [Solomon and Price (Pharmacoeconomics 11:1-10, 1997)]. Indirect costs associated with reduced productivity represent a substantial proportion of the total cost of migraine as well. Migraine has a major impact on the working sector of the population, and therefore, determining the indirect costs outweighs the direct costs. This study will explain the notion of cost of illness, examining how it could be applied in such a framework. Then, an overview of the studies aimed at measuring direct and indirect costs of migraine and headache disorders will be
\end{abstract}

F. S. Mennini $(\bowtie) \cdot$ L. Gitto

CEIS-Sanità (Centre for Health Economics

and Management - CHEM), Faculty of Economics,

University of Rome "Tor Vergata", Rome, Italy

e-mail: f.mennini@uniroma2.it

P. Martelletti

2nd School of Medicine, St Andrea Hospital,

Sapienza University of Rome, Rome, Italy carried out, later shifting on to the relationship between costs and quality of life for people affected by headache disorders. Finally, a brief review on advantages of new pharmaceuticals and preventive treatments for migraine for patients and society will outline improvements in the context of costeffectiveness and cost-utility analysis.

Keywords Migraine - Cost of illness - Disease costing Economic evaluation

\section{Introduction}

Migraine is a very common disorder, affecting about $11 \%$ of adult populations in Western countries. Migraine can be classified among cephalalgias, a kind of disease widespread in the population: the percentages of the adult population with active headache disorders are, globally, $46 \%$ for headache in general, $11 \%$ for migraine, $42 \%$ for tensiontype headache and $3 \%$ for chronic daily headache [25].

Prevalence of migraine is highest during the peak productive years-between 25 and 55 years of age.

The prevalence is higher in females than males at all post-pubertal ages, but the sex ratio varies with age [16]. On the World Health Organization's ranking of causes of disability, headache disorders are among the ten most disabling conditions for the two genders, and among the five most disabling conditions for women.

Hence, the impact of headache disorders is a problem of enormous proportions, both for individual and society. The medical literature tried to assess the effects on individuals, by examining prevalence, distribution, attack frequency and duration, and headache-related disability, as well as its effects on society, looking at the socio-economic burden of headache disorders $[14,22]$. 
The issue of costs represents an important problem too, concerning both direct and indirect cost. Direct costs concern mainly expenses for drugs.

Drugs are not often considered in an appropriate way: most of the people suffering from migraine, in fact, manage their headaches without conventional medical advice and generally treat their attacks with over-the-counter medication. This often gives rise to a high percentage of visits to different health professionals and, once the disease has become chronic, to a large number of prescriptions for medication, which in turn produces an increase in costs [27].

Migraine has a considerable impact on functional capacity, resulting in disrupted work and social activities: many migraineurs do not seek medical attention because they have not been accurately diagnosed by a physician or do not use prescribed medication [23]. Indirect costs associated with reduced productivity represent a substantial proportion of the total cost of migraine as well. Migraine has a major impact on the working sector of the population, and therefore, determining that indirect costs outweigh the direct costs.

It has been estimated that chronic headache is one of the most costly illnesses of modern society, on account of its widespread presence in industrialised countries [14].

During the last years, several studies on chronic headaches have further analysed the costs, stressing the negative influence of headache on quality-of-life parameters [4], including their influence on functioning ability or work ability.

Treatment with new drugs represents a new emerging therapeutic tool in the field of headache treatment, with promising application for chronic forms. Together with the appearance of new pharmaceuticals, the creation of specialised headache centres has to be mentioned, and the dissemination of programmes to educate general practitioners and other health care specialists in matters concerning headache, that where implemented, have led to positive results [27].

Since standardised measures to evaluate losses of utility due to migraine have not been properly developed so far, the issue of costs is still the most relevant: the approach of cost of illness is particularly well suited to analyse the burden of headache: moreover, it allows to correctly evaluate the extent of benefits (i.e. reduction of costs) that is possible to obtain from a preventive cure of migraine.

This study is organised as follows. The next section will explain the notion of cost of illness, examining how it could be applied in such a framework. Then, an overview of the studies aimed at measuring direct and indirect costs of migraine and headache disorders will be carried out, later shifting on to the relationship between costs and quality of life for people affected by headache disorders.
Finally, a brief review on advantages of new pharmaceuticals and preventive treatments for migraine for patients and society will outline improvements in the context of cost-effectiveness and cost-utility analysis.

\section{Cost of illness and headache}

"Cost of illness" represents economic evaluation methodology through which it is possible to determine the cost caused by illnesses on the population.

The principal aim of the economic evaluation method is to define explicit value of the human life and thereby, to determine the cost in terms of loss of productivity caused by death of an individual.

More recently, this approach has been used to calculate the social and economic costs, in terms of loss of productivity, caused by a disease that only in the most serious cases results in the death of an individual.

The production losses resulting from morbidity are reflected in work-time reductions that may be calculated through absence. Recent studies have demonstrated that, in EU countries, the loss of productivity caused by headache represents one of the most important problems.

The production losses, also defined as indirect costs, are not the only social costs caused by diseases. Drummond [7] pointed also to the negative variations that are reflected on the two fundamental quantities: consumption of resources and state of health.

The former is also defined as direct costs. Patients in primary care with headache cost at least $87 \%$ more than their similar-age and same gender counterparts without headache [8]. Patients with migraine exact as much as 1.6-fold higher overall costs in comparison to patients without migraine [5]. Direct costs include all the healthcare costs caused by a disease, from its diagnosis to the patient's treatment and rehabilitation. Hence, this means the clinical and instrumental analyses called for by a diagnosis, the drugs, as well as any other therapeutical measure (i.e. surgical operation, radiotherapy). The resources include the services provided by the health personnel (physicians, nurses and other workers) and that part of overhead costs imputable to the disease. Hospital costs universally represent only a very small portion of total migraine management costs [13].

These are the most easily identifiable costs. However, it is still not possible to precisely quantify the direct costs of migraine because there is general agreement that a high percentage of migraneurs never consult a physician for their illness - between 19\% and 44\% in the various studies - and only a small percentage (from 16\% to $36 \%$ ) regularly consult their physician. They may be differentiated depending on who provides the resources: the healthcare 
service or else the patients and their relatives. Such a distinction proves useful as it permits to detect the differences existing between disease costing - this being an economic appraisal method-and the financial analyses that only consider those costs that affect the year-end figures of a business. Indeed, economic appraisal is a method for evaluating social costs, regardless of whoever bears them.

The calculation of direct costs must also include a number of costs that are not related to healthcare, such as the time needed to get to the facilities providing healthcare services. They also include transportation costs, and waiting-time costs, provided that this time is not subtracted from the productive activity since, otherwise, this would lead to duplication where it would be also calculated among production losses. In the case of a few pathologies one must also include special diets, changes in the home in the case of a few home-delivered treatments, or in one's clothes, when a prosthesis or a different medical device is called for.

So, there are more problems connected to the cost of illness related to headache. It still appears as a method that can lead to different results in accordance with the utilized principle of evaluation.

We need to consider not only the social dimension of illness (headache), but the real possibility to reduce costs, thanks to existing technologies (medical devices, drugs etc...) too.

\section{Direct and indirect costs of migraine}

The financial burden of migraine has constituted the main issue of many analyses. Goldberg [11] has seen how migraine determines costs totaling as much as 17 billion dollars in the United States. Most of the costs are for outpatient services, such as medications, office or clinic visits, emergency department visits, laboratory and diagnostic services, and management of treatment side effects. Of the total annual cost associated with treatment of migraine, about one tenth ( $\$ 1.5$ billion) goes to medication, with triptans accounting for the majority of this amount (\$1.18 billion).

Triptans are selective 5-HT receptor agonists that are specific and effective treatments in the management of migraine: they meet the acute treatment goal of rapid relief with minimal side effects. Triptans are also associated with improved quality of life [6].

These agents are highly effective, but with a mean cost per prescription of $\$ 160$, they are also among the leading contributors to cost. When properly used, their clinical effectiveness justifies their cost. However, their cost is not definitive: regarding their use, the triptans are not interchangeable, and costs as well as clinical outcomes may vary with different agents in this class and according to patients' response.

Indirect costs, whose estimate is of more of 14.5 billion dollars (of which 7.9 billion dollars was due to absenteeism, 5.4 billion dollars to diminished productivity, and 1.2 billion dollars to medical costs), add substantially to the total [13, 24].

Emphasis on indirect costs can be seen in the study by Lipton et al. [17]: given that severe migraine can lead to disruption of work, family and social life, the direct costs of migraine, due to medical care, are small compared with the indirect costs caused by absence from work and reduced productivity.

The authors stress how population-based studies are required to assess incidence, prevalence and distribution of the disease. Studies already existing in literature, in fact, reveal that migraine is currently underdiagnosed and undertreated. Measures of severity may be useful as predictors of disability and healthcare use: moreover, such measures may also be useful in assessing the need for patient care and treatment and help target those more disabled by migraine. As a conclusion, the identification of patients who need more care and their treatment should reduce the impact of migraine on the individual and the burden of migraine on society.

In another study, Lipton et al. [18] stress once again the enormous health burden on individual and on society imposed by headache (the authors mention both headache and migraine). The condition affects about $18 \%$ of women and about $6 \%$ of men across their lifespan. Because its prevalence peaks during the most productive years, migraine is an important cause of lost work time. The focus of this study is just on work losses that are not uniformly distributed: the most disabled half of migraineurs account, in fact, for more than $80 \%$ of all work loss.

$\mathrm{Hu}$ et al. [13] attempt a comprehensive estimate of the financial burden of migraine in the US, considering mainly indirect costs. The authors look at several indicators:

- Bedridden days per year. Migraine-related disability was calculated as a function of the number of bedridden days in patients aged between 20 and 64 years based on results of a previous study (the Baltimore County Migraine Study). Subjects reported how often they needed bed rest when experiencing a migraine attack; response options were: never, rarely, less than $50 \%$ of the time, and more than $50 \%$ of the time; for calculations, these were translated into rates of 0,10 , 25 , and $75 \%$. An average percentage of attacks in which patients need bed rest (PAB) was generated for each age and sex stratum. Total number of bedridden days per year (BDY) in the given age and sex stratum was calculated as follows: 


$$
\mathrm{BDY}=\mathrm{NMS} \times \mathrm{FAY} \times \mathrm{PAB} \times \mathrm{ABH} / 24
$$

where NMS is the number of migraine sufferers in an age and sex stratum, FAY the frequency of migraine attacks per year, $\mathrm{ABH}$ the average bedridden hours when lying down with a migraine attack. By dividing by 24 , bedridden hours were converted into bedridden days.

- Health care resource use. The costs of health care resource utilisation associated with migraine were obtained from an analysis of 1994 data from MEDSTAT's MarketScan data set. This included both inpatient, outpatient, and prescription drug claims for employees and their dependents for more than 40 large employers in the United States. For outpatient encounters, either primary or secondary diagnoses were accepted. For hospitalizations, only the principal diagnosis was accepted. Migraine-related drug costs were estimated only for those patients who had at least one migraine-related medical encounter.

- Economic loss due to missed workdays. Missed workdays and impaired work performance were combined with data from the Bureau of Labor Statistics with respect to percentage of population working for pay and average working hours per week.

The total number of migraine-related missed workdays (TMWD) per year was calculated for each age- and sexspecific stratum as follows:

$\mathrm{TMWD}=\mathrm{NMS} \times \mathrm{MWD} \times \mathrm{PWP} \times \mathrm{WHW} / 40$

where MWD is average migraine-related missed workdays per year, PWP is the percentage of the population working for pay, estimated at $73 \%$ for males and $57 \%$ for females, WHW is the average working hours per week, most recently reported as 35 working hours for both sexes.

Divided by 40 , working hours have been converted into the standard fulltime level (8 working hours per day).

Impaired work performance was calculated as a function of the number of workdays with migraine (NWDM) and reduced work efficiency during the attacks. The NWDM was estimated as follows:

$\mathrm{NWDM}=\mathrm{NMS} \times \mathrm{WDM} \times \mathrm{PWP} \times \mathrm{WHW} / 40$.

The average number of workdays with migraine (WDM) per year was estimated based on patient self-report.

Lost workday equivalent (LWDE) due to impaired work performance was calculated as follows:

$\mathrm{LWDE}=\mathrm{NWDM} \times(1-\mathrm{EWM})$,

where EWM is the average effectiveness at work with migraine, estimated at $42 \%$ for men and $34 \%$ for women.

- Economic loss due to reduced productivity. The total employment lost due to migraine (TELM) in dollar terms was calculated as follows, assuming $8 \mathrm{~h}$ for each working day:

TELM $=($ TMWD + LWDE $) \times$ hourly salary $\times 8$.

The analysis put in evidence what extent patients with migraine are frequently disabled during their acute attacks. Although migraine-related disability can be reflected by both bedridden days and restricted activities, the authors emphasized bedridden days because they can be more reliably reported and quantified. About one-third of migraine sufferers experienced severe disability or the need for bed rest following attacks, and an additional 50\% reported mild or moderate disability. However, the overall estimates still do not capture several important components of the burden of disease. There is room for further analyses that should be carried out bearing in mind that direct costs represent a small proportion of the overall societal costs of migraine (therefore analyses should concentrate on indirect costs).

Nevertheless, there are a few reasons to believe that even the direct costs have been underestimated. This conclusion derives by considering that medical claims do not capture all migraine-related treatment costs because the disease is often not treated with specific therapies. Moreover, it is quite complicated to measure over-the-counter or preventive medications and nondrug-related interventions.

Indirect costs are the main content of a recent study by Hawkins et al. [12]: to estimate the indirect burden of illness of migraine, the authors compared the average annual indirect expenditures of a group of employees with migraine with a matched group of employees without migraine (control group). The burden of illness of migraine was defined as the difference in average indirect expenditures per person between migraine and control cohorts. Indirect cost components included in this study were workplace absence, short-term disability and workers' compensation claims.

Controls were matched to the migraine cohort according to the predicted probability of having a migraine; this probability was estimated for each patient on the basis of a logistic regression analysis of having a migraine that controlled for demographics (age, gender, region, location, year, and type of insurance) and overall comorbidities.

A second-stage regression was used to estimate the indirect burden of migraine. Specifically, the second-stage regression used total indirect expenditures as the dependent variable and the same independent variables used in the propensity score matching, plus a dummy indicator to denote migraine patients. The second-stage regression estimated by applying a generalized linear model (GLM), controlled for any remaining differences between the cohorts after matching.

It was estimated that employees with migraine cost employers approximately $\$ 12$ billion per year due to 
absenteeism, short-term disability, and/or workers' compensation claims. Most of the indirect migraine expenditures were driven by absenteeism. Results of the study confirm that the overall burden of migraine on society is large.

In a cross-study comparison, the estimated peremployee absenteeism plus short term disability expenditures for migraine in this study were shown to be greater than those of other common conditions such as chronic obstructive pulmonary disorder, heart disease, depression, chronic renal failure, diabetes, and asthma.

Compared with the study by $\mathrm{Hu}$ et al., which found absenteeism costs to be about $\$ 8$ billion ( $\$ 10.1$ billion 2004 dollars), the estimate of absence cost from the current study (\$9 billion), which used a different methodological approach was consistent although total indirect burden of illness estimated by Hawkins et al. cannot be directly compared with the estimate from $\mathrm{Hu}$ et al., as the two studies captured different sets of indirect cost components: specifically, Hu et al. did not include short-term disability and workers' claims.

These results are very important when viewed in terms of the loss of production capacity (debility) that occurs when a sick individual goes to work-presenteeism: an individual suffering from migraine will not show the same level of productivity as a non-suffering individual.

Some authors have shown how presenteeism was the most important driver of overall costs, and how its impact was greatest in patients with migraine/headache, accounting for $89 \%$ of the total cost burden [10].

The authors conclude their study mentioning the therapeutic opportunities for headache and potential advantages: higher direct costs, due to more expensive drugs, could be offset by savings in indirect costs (i.e. reduced and lost productivity).

Other international studies have estimated the burden of migraine: Bigal et al. [3] have conducted such an analysis for Brazil, implementing both a cost-analysis and a costeffectiveness analysis. The objective of their study is to address national health expenses in the most efficient way. Data refer to 1999 and distinguish among Brazilian demographic characteristics, characteristics of the public health system related to primary, secondary, and tertiary care, the last being subdivided into emergency department and hospital care, and estimation of the number of migraine consultations at each complexity level.

The authors stress how in Brazil, migraineurs seen in the public health system most often are discharged with an acute treatment, usually a non-specific medication: this way of intervention is compared with a proposed stratified care model that uses triptan as an acute care medication.

The annual costs of the treatment were calculated according to the following equation:
$\mathrm{AC}=\mathrm{P} \times \mathrm{N} \times \mathrm{C}+\mathrm{P} \times \mathrm{Cp}+\mathrm{P} \times \mathrm{Cat} \times \mathrm{AMA}$,

where $\mathrm{P}$ is the number of patients, $\mathrm{N}$ is the number of consultations per patient, $\mathrm{C}$ is the cost of consultation per level, $\mathrm{Cp}$ is the cost of preventive drugs, Cat is the cost of acute therapy drugs, AMA is the number of migraine attacks per year, and produced the following results: the estimated cost of a consultation for migraine on the primary care level was US $\$ 11.53$; on the secondary care level, US \$22.18; in the emergency department, \$34.82; and for hospitalization, US $\$ 217.93$. Hence, the total estimated public health system expenses for migraine were US $\$ 140388469.60$. The model proposed by authors that include specific acute migraine therapies, would imply a cost reduction of $6.2 \%$ (US $\$ 7514604.40$ ), improving, at the same time, the level of quality of care of the public health system.

Recently, Vinding et al. [26] have estimated the burden of headache in a patient population from a specialized headache centre in Denmark. The sample was made of 55 patients (12 males and 43 females) whose median headache frequency was of 15 days/month; information was collected through structured interviews, prospective headache diaries and standardized self-administered questionnaires. The interview contained a total of 116 questions about the socio-economic impact of headache disorders. The interview included an extensive description of the influence on working ability, personal impact, utilization of health services and medicine, and was conducted by a trained medical student blinded to the remaining information about headache diagnosis, frequency and medication use.

Very high utilization of the healthcare system and a high absence rate due to headache of 12 days/year were reported. Eighty-one percent of patients experienced a marked decrease in work effectiveness. Overall, $91 \%$ felt hampered by their headache on a daily basis and $98 \%$ had had expenses for headache medication. The absence rate reported by the study was highly skewed as $19 \%$ (nine patients) were $>60$ days absent from work due to headache and of those, $10 \%(n=5)$ had been absent a full year. Due to headache, $90 \%$ (43/48) had been absent from work at least once and due to all causes $96 \%$ (47/49) had had at least one absent day during the last year.

Further specification could be added to analyses when estimating the burden of headache according to different levels of pain. Auray [2] takes the classification made by the International Headache Society that distinguishes three categories for headache. The author carried out an extensive survey on a sample of 10585 individuals in the French adult population (data of 1999). The results showed a prevalence of $17.3 \%$ for migraine and nearly $30 \%$ for headaches. The average expenditure for a headache patient is about 220 Euro (10\% for GP consultations, $11 \%$ for 
laboratory evaluations, $17 \%$ for specialist consultations, $18 \%$ for drugs and $44 \%$ for hospital costs). However, this partition of cost depends largely on the headache category: in the same way, it could be concluded that, although the most acute headaches lead to the most severe deterioration of quality of life, professional or school activities are not affected similarly.

Overall, it could be concluded that costs, both direct and indirect, are unknown: it is now established that the indirect costs of migraine outweigh the direct costs and therefore represent an obvious target for healthcare intervention, aimed at reducing the impact of migraine [23]: these conclusions come from a study carried out in 1997, but they could be well replicated today.

\section{Migraine and quality of life}

A different approach followed by more recent studies is based on quality of life rather than economic evaluation.

Despite the prevalence and substantial economic burden of migraine, no standardized measures of quality of care exist.

Gagne et al. [9], in a study aimed at drawing a comprehensive review for quality of care measures, stress how no standardized measures for measuring quality of life for patients suffering from migraine have been developed so far. For example, the Health Plan Employer Data and Information Set, maintained by the National Committee for Quality Assurance is widely used in the US to assess quality of care at the health-plan level, but it does not include any headache, or migraine-related measures. This may be due, in part, to a lack of understanding of migraine or its underdiagnosis. For example, migraine was not even included in the first Global Burden of Disease in 1990 [15]. The American Migraine Prevalence and Prevention Study found that only $56.2 \%$ of those with migraine had ever received a medical diagnosis.

Since many publications provide insights into the large clinical and economic burden of the disorder, patients, employers, disease management providers, and health plans alike would benefit from a standardized set of migraine quality measures.

A review about the existing quality of care measures for migraine is carried out by Gagne et al. [9]: such measures include patient-reported measures and non-patient reported, diagnosis-related, prevention-related, and treatmentrelated indicators.

Most existing measures have been developed by the Institute for Clinical Systems Improvement or summarized and reported by the RAND Corporation. However, few migraine care quality measures exist, and those that are available are not easily implemented at the health-plan level. Hence, significant effort is still needed to determine what and how to measure quality among health plans to improve the quality of care delivered to individuals with migraine.

Leonardi et al. [15] summarize classification for disability due to headache according to WHO classification: using disability-adjusted life years as a summary measure of population health (which adds disability to mortality), WHO has shown that mental and neurological disorders collectively account for $30.8 \%$ of all years of healthy life lost to disability whilst migraine; one amongst these, alone accounts for $1.4 \%$ and is among the top 20 causes of disability worldwide. This information is combined with the increasing widely accepted belief that disability and functioning are relevant parameters for monitoring the health of nations and that there is an increasing need to measure them.

Classification of functioning, disability and health applied to headache disorders allows comparability with other health conditions as well as evaluation of the role of the environment as a cause of disability amongst people with headache.

\section{Treatment of migraine with new drugs and improvements in terms of cost savings and quality of life}

Appropriate treatment of migraine can, of course, decrease the level of costs [6]. Given the level of costs, likely to interest such a high percentage of population affected by migraine, prevention, early intervention or effective treatment strategies for headache disorders may be highly cost effective.

Cost-effective models can be used to understand the effect of treatment choices on health care budgets. All aspects of effectiveness (efficacy, tolerability, and cost) should be considered to reduce overall managed care expenditures for migraine treatment.

The choice of a new or an old therapeutic treatment depends on a number of factors. Moreover, the evaluation of the cost, in terms of economics, but also in terms of quality of life and type of facilities necessary for one treatment are crucial criteria. Treatment with new drugs represents a new emerging therapeutic tool in the field of headache treatment, with promising application for chronic forms.

In evaluating treatment strategies not only the activity of a drug in reaching the main end-points, (i.e. pain free or headache relief) should be considered, but also the safety and perception of safety by patients, and the cost effectiveness, including indirect costs compared with personal and social benefits. There is no evidence of cost-benefit 
analysis as suggested by Pini et al. [21]. Rather, there are several cost-effectiveness studies aimed at evaluating costeffectiveness ratios for triptans or other drugs [4, 28].

Migraine prophylaxis is aimed at preventing frequent attacks and the development of a long-term condition that often incurs heavy costs for abortive treatment, diagnostic services, and medical care. Agents approved for migraine prophylaxis include the antiepileptics divalproex and topiramate and the beta blockers propranolol and timolol. As with abortive therapy, costs vary widely among these prophylactic agents.

A new approach to migraine prophylaxis is injection of botulinum toxin [19]. Overall, positive results have been reported concerning the reduction of the duration and the intensity of the attacks, as well as the muscle contraction.

Prophylactic migraine treatment effectiveness has been evaluated by many studies: it is possible to mention, for example, the article by Adelman and Von Seggern [1] to see how issues concerning advantages of preventive treatment had already been considered more than a decade ago.

An individual approach is, however, required: Pierangeli et al. [20] warn about the need to weigh the potential benefits against the adverse effects associated with each agent in determining the optimal preventive regimen for individual patients considering any co-morbid condition.

Moreover, an important role has to be played by the physician as regarding the decision to treat and the choice of prophylactic drug that should be taken with the patient. What is important is to balance expectations and therapeutic realities for each drug.

\section{Conclusion}

By way of conclusion, it should be recalled that in the headache field we are far behind other pathologies, where there is a greater knowledge of the economic aspects of both the pathology-related costs and the likely benefits resulting from different therapeutical approaches.

It may be noted that there are still many unsolved problems in disease costing, to the point that it still appears as a set of method that may lead to extremely different outcomes depending on the evaluation approach being used.

In any event, it should be noted that, in this context, one needs to take into account not only the social cost of a given disease, but also the real possibilities of cutting down those costs thanks to the existing technologies, meaning the diagnostic, therapeutic and rehabilitative tools that are already available or are being adopted.

Notwithstanding the disease costing problems, which are also reflected in the cost-benefit approaches, it is important for the economic analysis to gain ground since there is a growing need to keep account of the available resources and the results attainable in the healthcare policies, from the central to the peripheral levels, where the above-referred evaluation tools referred prove even more expedient.

Moreover, given the social relevance of migraine, it should be important to increase the knowledge related to the economic consequences of prevention through an increase of availability of health service.

From the analysis of prevalence, incidence, morbidity and consequence of the state of health caused by headache, it seems important to awaken the scientific community and policy makers to the problems connected to the economic costs of headache and how it should be faced using the implementation of specified "observatory."

For that reason it is essential to increase economic evaluation studies to be able to estimate economic and financial costs of headache (i.e. see The Global Campaign to Reduce the Burden of Headache Worldwide-WHO).

Acknowledgments This Tutorial partly results from the lesson of the Author (FSM) at the Master in Headache Medicine, Sapienza University of Rome, during the academic year 2007-2008 (http://w3.uniroma1.it/headache). This Academic Educational activity is done under the supervision of the WHO Global Campaign to Reduce the Burden of Headache Worldwide "Lifting The Burden"-Working Group for Specialist Education (SEWG) and Working Group for Outcome Measures (OMWG) (http://www.l-t-b.org).

\section{Conflict of interest None.}

\section{References}

1. Adelman JU, Von Seggern R (1995) Cost considerations in headache treatment. Part 1: prophylactic migraine treatment. Headache 35(8):479-487

2. Auray JP (2006) Socio-economic impact of migraine and headaches in France. CNS Drugs 20(1):37-46

3. Bigal ME, Rapoport AM, Bordini CA, Tepper SJ, Shftell FD, Speciali JG (2003) Burden of migraine in Brazil: estimate of cost of migraine to the public health system and an analytical study of the cost-effectiveness of a stratified model of care. Headache 43(7):742-754

4. Blumenfeld A (2003) Botulinum toxin type A treatment of disabling migraine headache: a randomised double-blind, placebocontrolled study. Headache 43:853-860

5. Clouse JC, Osterbaus JT (1994) Healthcare resource use and costs associated with migraine in a managed healthcare setting. Ann Pharmacother 28(5):659-664

6. Dodick DW, Lipsy RJ (2004) Advances in migraine management: implications for managed care organizations. Manag Care 13(5):45-51

7. Drummond M (1980) Principles of economic appraisal in health care, Oxford University Press

8. Fishman P, Von Korff M, Lozano P et al (1997) Chronic care costs in managed care. Health Aff 16(3):239-247

9. Gagne JJ, Leas B, Lofland JH, Goldfarb N, Freitag F, Silberstein S (2007) Quality of care measures for migraine: a comprehensive review. Dis Manag 10(3):138-146 
10. Goetzel RZ, Long SR, Ozminkowski RJ, Hawkins K, Wang S, Lynch W (2004) Health, absence, disability, and presenteeism cost estimates of certain physical and mental health conditions affecting US employers. J Occup Environ Med 46:398-412

11. Goldberg LD (2005) The cost of migraine and its treatment. Am J Manag Care 11(2 Suppl):S62-S67

12. Hawkins K, Wang S, Rupnow MF (2007) Indirect cost burden of migraine in the United States. J Occup Environ Med 49(4):368374

13. Hu XH, Markson LE, Lipton RB, Stewart WF, Berger ML (1999) Burden of migraine in the United States: disability and economic costs. Arch Intern Med 159(8):813-818

14. Lanteri-Minet $M$ et al (2003) Prevalence and description of chronic daily headache in the general population in France. Pain 102:143-149

15. Leonardi M, Steiner TJ, Scher AT, Lipton RB (2005) The global burden of migraine: measuring disability in headache disorders with WHO's classification of functioning, disability and health (ICF). J Headache Pain 6(6):429-440

16. Lipton RB, Stewart WF, Scher AI (2001) Epidemiology and economic impact of migraine. Curr Med Res Opin 17(Suppl 1):S4-S12

17. Lipton RB, Stewart WF, Von Korff M (1994) The burden of migraine. A review of cost to society. Pharmacoeconomics 6(3):215-221

18. Lipton RB, Stewart WF, Von Korff M (1997) Burden of migraine: societal costs and therapeutic opportunities. Neurology 48(3 Suppl. 3):S4-S9

19. Mennini FS, Fioravanti L, Piasini L, Palazzo F, Coloprisco G, Martelletti P (2004) A one-year economic evaluation of botulinum toxin type a treatment of chronic tension-type headaches: part I. J Headache Pain

20. Pierangeli G, Cevoli S, Sancisi E, Grimaldi D, Zanigni S, Montagna P, Cortelli P (2006) Which therapy for which patient? Neurol Sci 27(Suppl. 2):S153-S158

21. Pini LA, Cainazzo MM, Brovia D (2005) Risk benefit and costbenefit ratio in headache treatment. J Headache Pain 6(4):315318

22. Rasmussen BK (1999) Epidemiology and socio-economic impact of headache. Cephalalgia 19(Suppl. 25):20-23

23. Solomon GD, Price KL (1997) Burden of migraine. A review of its socioeconomic impact. Pharmacoeconomics 11(Suppl. 1):110

24. Stewart WF, Ricci JA, Chee E, Morganstein D, Lipton R (2003) Lost productive time and cost due to common pain conditions in the US workforce. JAMA 290:2443-2454

25. Stovner LJ, Hagen K, Jensen R, Katsarava Z, Lipton R, Scher A, Steiner T, Zwart JA (2007) The global burden of headache: a documentation of headache prevalence and disability worldwide. Cephalalgia 27(3):193-210

26. Vinding GR, Zeeberg P, Lyngberg A, Nielsen RT, Jensen R (2007) The burden of headache in a patient population from a specialized headache centre. Cephalalgia 27(3):263-270

27. Volcy-Gomez M (2006) The impact of migraine and other primary headaches on the health system and in social and economic terms. Rev Neurol 43(4):228-235

28. Wheeler AH (1998) Botulinum toxin A, adjunctive therapy for refractory headaches associated with pericranial muscle tension. Headache 38(6):468-471 